\title{
Notes on Operations GMD or No GMD \\ RDA Implementation for a Consortial Catalog
}

\author{
James Kalwara, Melody Dale, and Marty Coleman
}

This paper explores the benefits of establishing item-specific terms for General Material Designations (GMDs) for library consortia implementing Resource Description and Access (RDA). While RDA includes a new approach towards the description and categorization of an item's physical medium through the assignment of content, media, and carrier types (CMCs), thus replacing the GMD, libraries may still benefit from GMD retention in their online catalogs to help support user tasks and help contextualize CMC information. This paper presents the challenges that Mississippi State University Libraries experienced in leading RDA enrichment for the Mississippi Library Partnership (MLP) consortium. Additionally, it discusses parameters for libraries to consider when working with a vendor for RDA enrichment in a consortial environment.

James Kalwara (james.kalwara@colo rado.edu) is a Monographic Cataloger at the University of Colorado Boulder. Melody Dale (mdale@library.msstate .edu) is an Education Librarian at Mississippi State University. Marty Coleman (mcoleman@library.msstate.edu) is an Acquisitions Librarian at Mississippi State University.

Manuscript submitted May 25, 2016: returned to authors for revision September 2, 2016; revised manuscript submitted October 30, 2016; manuscript returned to authors for minor revision January 4, 2017; revised manuscript submitted February 3, 2017; accepted for publication March 31, 2017.

This paper is based on a presentation that was delivered at the Southeastern SirsiDynix Regional Users Group Conference on Tuesday, August 4, 2015 at Mississippi State University and also at the Association of Library Collections and Technical Services Catalog Management Interest Group Meeting at the American Library Association Midwinter Meeting on Saturday, January 9, 2016 at the Boston Convention \& Exhibition Center.

The authors would like to thank Anita Winger at Mississippi State University for supplying vital data and timelines for this paper.
$\mathrm{T}$ he Library of Congress's implementation of RDA in March 2013 prompted many libraries to reassess their local cataloging practices. A major change with RDA was the change from the General Material Designation (GMD) to the content, media, and carrier types (CMCs) provided in MARC 336, 337, and 338 (commonly referred to as 33X) fields. ${ }^{1}$ In 2010 , a librarian voiced concern at the loss of GMDs when addressing a columnist: "Dear Elsie, Is it true that the GMD will disappear with RDA? If so, how will we alert our patrons, and ourselves, to the fact that a title is a CD, a DVD, and so on? Designated, and would like to stay that way, in Decatur."2 At that time, Mississippi State University's (MSU) catalogers shared the same concern and began taking steps to develop training and implementation plans for this new cataloging standard while considering the future of GMDs in the consortial catalog.

During RDA training, MSU's catalogers discussed display and indexing decisions for RDA elements and whether the GMD would remain useful with the standard's new rules. Catalogers agreed that GMDs contextualize 33X terms and clearly differentiate materials that share the same title. After discussing this concern with various library departments and consortial partners, MSU's catalogers determined that retaining GMDs remained essential to supporting resource discoverability. However, catalogers would need to update legacy GMD terms by selecting more item-specific terms to better support user tasks. Members of several departments in MSU Libraries suggested using "common terms" in place of GMDs for their local bibliographic records to support patron search behaviors in the consortial catalog. For instance, the common term "DVD" would in some cases replace the GMD "videorecording," and similarly, the common term "MP3" would replace the GMD "electronic resource" in some cases.

The decision to implement RDA and to retain GMDs affected the MLP, which is comprised of fifty-four libraries with distinct user needs. As several consortial libraries already used non-standard common terms locally in place of GMDs, a collective decision was reached to continue this practice to establish consistent metadata across the catalog while proceeding with RDA enrichment. 
MLP libraries agreed to use the established common terms and to incorporate RDA elements and practices into nonRDA records, including spelling out abbreviations found in the MARC 300 and 504 fields, adding the 33X fields, and converting the 260 field to appropriate 264 fields, to maintain consistency. Additionally, MSU Libraries agreed to provide training and documentation to MLP members as needed.

\section{Literature Review}

This literature review explores both the historical and the current climate of GMD usage in library catalogs and how libraries have respectively handled GMD replacement and CMC inclusion following RDA implementation. GMDs originated from the necessity to distinguish between different material types with the same title. For instance, the GMD "videorecording" distinguishes a title's medium from other possible manifestations of the same title, including a sound recording or an electronic resource.

Initially, media and print materials were housed in separate catalogs; however, in some libraries, media items were uncataloged and simply stored in particular workrooms. ${ }^{3}$ In the 1960s, libraries recognized the advantages of providing bibliographic records for all material types within a unified catalog. ${ }^{4}$ Libraries began using media codes, which were later renamed media designators, to identify non-print materials. ${ }^{5}$ The Anglo-American Cataloging Rules First Edition (AACR) standardized a small vocabulary of media designators; however, they were not applied to all types of materials, nor were all media types included in the code. ${ }^{6}$ Media designation was renamed to "general material designation" with the second edition of AACR (AACR2), which strategically placed the GMD directly after the title proper to notify the user of an item's physical medium. ${ }^{7}$

While GMDs remain beneficial for users, there are also limitations to their usefulness. Caudle and Schmitz discovered that patrons sought more detailed information regarding a resource's format type than what was presented by the GMD. ${ }^{8}$ GMDs also do not consistently communicate an item's mode of issuance or carrier information. ${ }^{9}$ For example, the GMD "filmstrip" represents only one physical format; whereas the GMD "sound recording" can represent multiple carrier types, including audio cassettes, compact discs, or audiotape reels. Schmitz argued that providing the mode of issuance and carrier type information for an electronic resource enables users to clearly distinguish between a newspaper and an electronic book, or to clarify whether an audio disc is a music CD or a vinyl record. ${ }^{10}$ Oliver indicated that GMDs inconsistently describe an item's physical medium since they represent the attributes of an item on a work, expression, and manifestation level but inadequately provide description on an item level. ${ }^{11}$ Ou and Saxon reiterated the shortcomings of GMDs' capacity for item-level description and categorization by illustrating that while the GMD "electronic resource" describes a resource's carrier type, the same resource could also be assigned the GMD "cartographic material," which describes the resource's content type. ${ }^{12}$ Furthermore, a motion picture may be assigned the GMD "videorecording," yet when the same title is issued as a streaming video, the GMD "electronic resource" is assigned since that is considered as the primary medium. ${ }^{13}$ Additionally, Seikel and Steele suggested that GMDs have become irrelevant with user search patterns, due to updates to terms such as "sound cassette" and "videodisc," which have been superseded by the more commonly used terms "audio tape" and "DVD."14

RDA seeks to address and remedy the GMD's limitations and issues by replacing them with CMCs, which are provided in the MARC 336 Content Type, 337 Media Type, and 338 Carrier Type fields. ${ }^{15}$ The content type is the form of communication through which a work is expressed. ${ }^{16}$ The media type reflects the general type of intermediation device required to view, play, run, or access the content of a resource ${ }^{17}$ The carrier type reflects the format of the storage medium and housing of a carrier in combination with media type. ${ }^{18}$ Bernstein suggests that implementing CMCs allows for a more hierarchical structure for categorizing resources that addresses the complexities found in categorizing nonprint materials. ${ }^{19}$

While RDA takes a more granular approach to resource description, online public access catalogs (OPACs) and discovery systems are still developing functions to fully support RDA's practical applications. RDA's theoretical foundation is based on the Functional Requirements for Bibliographic Records (FRBR), which focuses on representing entities, attributes, and relationships. ${ }^{20}$ However, in 2011, the MARC format had incorporated relatively few developments that could take full advantage of the FRBR model. ${ }^{21}$ Since then, the MARC environment and integrated library systems (ILS) remain under development. Cronin illustrated that libraries had varying degrees of control over the indexing and record display options of their ILS. ${ }^{22}$ Historically, many institutions have had success in displaying CMC information through open-source software and cultivating support from their systems departments. Currently, major ILS systems offer CMC display options enabling libraries to choose which CMC information to display based on patron needs. By using an open-source online catalog, catalogers and library systems associates at Auburn University customized display functions necessary to display CMC information in their online catalog. ${ }^{23}$ Panchyshyn additionally proposed an innovative OPAC solution to commercial online catalogs by combining the item type icon with the RDA carrier type data from a bibliographic record. ${ }^{24}$ 
RDA conversion and enrichment have recently been prominent in the library literature as more libraries have implemented RDA. Panchyshyn and Park concluded that RDA enrichment is a necessary step to enhance legacy bibliographic metadata, which ultimately improves patron experience in the online catalog. ${ }^{25}$ Guajardo and Carlstone described their RDA enrichment procedure, including the addition of material type codes, which served to replace GMDs. ${ }^{26}$ While no uniform resolution can correct OPAC display issues, libraries strive to support user tasks by developing their own solutions. Until OPAC and bibliographic systems can fully support all theoretical aspects of RDA, many libraries will continue working towards creating a positive user experience by modifying aspects of national cataloging practices to best support their local needs.

\section{Case Study}

MSU Libraries leads cataloging efforts for fifty-four libraries within its statewide consortium, the MLP. Many libraries within the consortium lack adequate staffing and resources to undergo a catalog enrichment project. MSU Libraries' cataloging department includes staff members who provide original and complex cataloging for serials and monographs. Since it is fully staffed, MSU Libraries was well suited to lead RDA enrichment for the consortial catalog and to establish RDA cataloging procedures for the MLP.

\section{MSU Libraries' Trajectory to RDA}

Formal discussions regarding MSU Libraries' RDA implementation began in spring 2010 with catalogers tracking the trend via discussion lists. Following various RDA discussion list threads gave MSU's catalogers the opportunity to learn how similar institutions were planning RDA implementation. MSU's catalogers also followed LC's efforts, which began transitioning to RDA in June 2011, with full implementation in March 2013. MSU catalogers realized that in a consortial environment, RDA implementation was not limited to their own cataloging workflows and would also impact MLP's original cataloging practices. RDA was a major discussion topic at the 2013 and 2014 Southeastern SirsiDynix Regional Users Group Conferences (SERUG) where MSU cataloging and systems librarians and the MLP staff members discussed concerns about omitting GMDs when bibliographic records were enriched to incorporate RDA elements and practices.

As members of the Name Authority Cooperative Program (NACO) since 2002, MSU catalogers understood that they would have to incorporate RDA practices into the authority records that they contributed to the LC/NACO Authority File. From July 2011 to November 2012, MSU catalogers received training from an LC representative in creating personal name, corporate body, and series authority records using RDA. After completing RDA NACO training in November 2012, catalogers were ready to implement RDA authority control practices at MSU Libraries. The next step in MSU Libraries' RDA planning included training in creating bibliographic records using RDA.

In September 2013, MSU Libraries applied for OCLC "Enhance" status, which involved a training and review period with an LC representative. This period allowed them the opportunity to create original bibliographic records that were sent to a reviewer who provided feedback prior to contributing master records to WorldCat. Following the review period, in late 2013, MSU Libraries received "Enhance" status, which then presented the opportunity to apply to the LC Monographic Bibliographic Record Cooperative Program (BIBCO). After completing four webinars and training with an LC representative to learn how to create original RDA BIBCO bibliographic records and how to enhance non-RDA bibliographic records to BIBCO status, MSU Libraries were granted BIBCO authorization in April 2014.

By gaining independence to contribute RDA BIBCO bibliographic and NACO authority records, MSU catalogers demonstrated that they were prepared to implement RDA policy standards both at MSU Libraries and to their MLP partners. In addition to establishing RDA standards policy documentation for MLP's original and copy cataloging procedures, an important component of the proposed RDA implementation was enriching bibliographic records in the online catalog to incorporate RDA elements, thus hybridizing its catalog. This enrichment entailed adding RDA elements including the 33X fields, spelling out abbreviations in the 300 and 504 fields, and converting the publication statement from the 260 to 264 field in all bibliographic records in the consortial catalog. By enriching its records with the aforementioned RDA elements, the consortial catalog would provide clean and consistent metadata for its users. Planning for RDA enrichment and implementation additionally prompted MSU Libraries and the MLP to reassess its vocabulary of GMDs and discuss their retention in the catalog.

\section{Retaining GMDs}

In addition to MSU's cataloging unit, several committees were involved in the discussion of GMD retention, including MSU's Library Technologies Committee, the OPAC subcommittee, the Library Administrative Council, and an ad-hoc committee consisting of MLP and MSU Libraries' personnel. These committees collaborated in the decisionmaking process, concluding that retaining GMDs throughout RDA enrichment in the consortial catalog would best support user tasks and establish consistent metadata for 


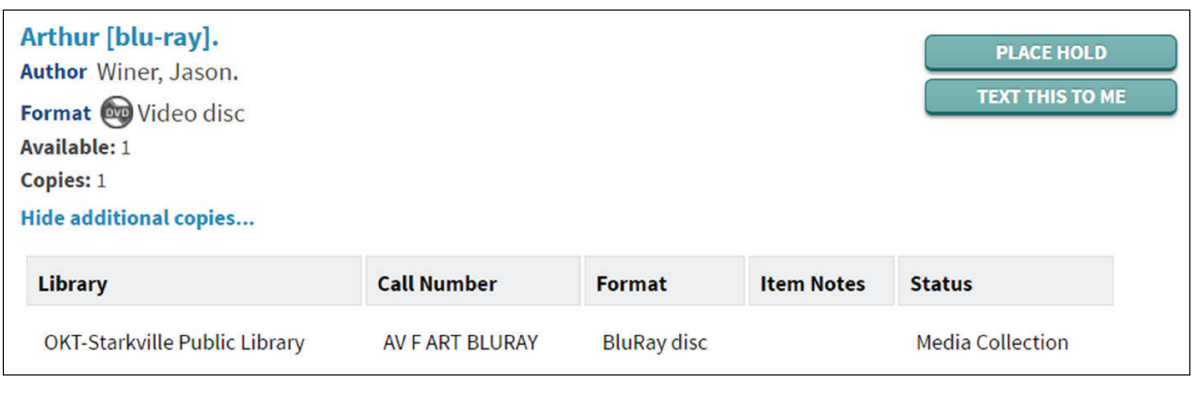

Figure 1. Example of GMD that Clarifies Format Icon Information

terms that might improve description of various types of electronic resources in the online catalog.

MSU catalogers felt strongly about retaining GMDs to support their workflows, specifically when performing routine database maintenance and copy cataloging procedures, such as differentiating between media and non-media titles published in multiple formats. Establishing consistent and clean metadata for the

user convenience. MSU's cataloging and computer systems departments moved forward with RDA enrichment and implementation.

\section{RDA Implementation}

In April 2014, Backstage Library Works (Backstage) approached MSU Libraries to serve as a testbed institution for vendor-supplied RDA enrichment. Backstage provides the benefit of establishing a customized RDA Profile, allowing an institution to define MARC data element parameters for RDA enrichment. To establish an RDA Profile for MSU Libraries, coding options for legacy bibliographic and authority records in the online catalog were explored to reflect specific RDA elements. By creating a customized RDA Profile for bibliographic record validation and authority record cleanup, MSU Libraries established preferences used in creating an algorithm to generate newly revised GMDs, replace the 260 with 264 fields, add 33X fields, and spell out abbreviations in the 300 and 504 fields of bibliographic records for subsequent quarterly batch-record loading.

Feedback on user information seeking behaviors was compiled from discussions with public services librarians. The cataloging department met informally with staff members from the MSU Libraries' Research Services Department from May to June 2014 to gain broader perspectives on the usage of GMDs. Research Services librarians indicated that patrons, research librarians, and support staff relied heavily on GMDs to support searching, identifying, and in some cases selecting resources of interest. They concluded that GMDs were most useful in instances when the GMD differed from the icon used to display an item's format type.

Figure 1 provides an example of how a GMD may be useful in identifying an item's format despite ILS display limitations. In this case, the only icon displayed in the ILS is for a DVD. With the GMD's presence, patrons can more easily interpret and confirm information describing the item's format as a Blu-ray disc. Catalogers also met with MSU's electronic resources personnel to discuss expanding catalog was another major concern in deciding to retain GMDs. After receiving feedback from various departments and cataloger recommendations, MSU Libraries concluded that retaining GMDs would best establish clean metadata for the consortial catalog and best support user tasks of searching, identifying, and selecting when conducting basic or advanced search queries in the online catalog. However, after reviewing previously used GMDs, it became apparent to MSU and MLP catalogers that revising GMDs would maximize their usefulness and enhance the user experience.

\section{GMD Expansion and Updating the Catalog's Existing GMDs}

The first step in revising GMDs was to review a list of "common terms" from Backstage, presented in figure 2, that could appropriately replace GMDs. ${ }^{27}$

While some of the listed "common terms" were already being used by a handful of consortial libraries, many, including MSU Libraries, used AACR2's GMDs for cataloging practices. After reviewing the list of Backstage "common terms" and AACR2 GMDs, MSU catalogers identified outdated GMDs that could be revised or omitted. ${ }^{28}$ To do this, catalogers discussed each common term from the Backstage list designated for a particular format type. For example, figure 3 displays four possible options, found in green boxes, which represent a DVD video, including "DVD," "videodisc (DVD)," "videodisc," and "videorecording (DVD)." After discussing the clarity of each option, catalogers concluded "DVD" was the clearest term that would best support user needs.

To ensure that GMDs appropriately applied to their corresponding item type in an item record, catalogers mapped them to all known item types from the catalog, and then determined how they could be separated and enhanced for clarity (see hdl.handle.net/11668/13644). For instance, every item type that corresponded to the GMD "videorecording" was recorded in an Excel spreadsheet. These included "BLURAY," "DVD," "DVD-SET," "DVD-ROM," "VIDEO," "NF-AV," and "VHS." Catalogers devised new "common term" GMDs to include "Blu-ray," "DVD," "electronic 


\begin{tabular}{|lll|}
\hline activity card & globe & record \\
art original & government document & serial \\
art reproduction & graphic & slide set \\
atlas & kit & sound recording \\
audiocassette & large print & sound recording (cassette) \\
braille & laser disc & sound recording (compact disc) \\
cartographic chart & LP & sound recording (CD) \\
cartographic material & manuscript & sound recording (LP) \\
cartographic material (tactile) & map & study print \\
CD recording & map (tactile) & technical drawing \\
CD-ROM & microfiche & text \\
CDV & microfilm & text (large print) \\
chart & microform & toy \\
chart (large print) & microopaque & transparency \\
compact disc & microprint & US document \\
diorama & microscope slide & VHS \\
DVD & model & video CD \\
DVD-ROM & motion picture & video single disc \\
electronic resource & music & videocassette \\
electronic resource (CD-ROM) & music (braille) & videodisc \\
federal document & newspaper & videodisc (DVD) \\
filmstrip & periodical & videorecording \\
flash card & photograph & videorecording (DVD) \\
floppy & picture & videorecording (VHS) \\
game & realia & VSD \\
\hline
\end{tabular}

Figure 2. Backstage "Common Terms" List

video," "nonfiction audiovisual," and "VHS" that would be used to replace each previous instance of the GMD "videorecording." Referencing the Backstage table helped catalogers to create granular and item-specific GMDs. For example, when prompted to derive a new GMD for "DVDROM," catalogers concluded that "DVD-ROM (computer only)" would provide clear information about both the item's carrier and media type to contextualize the RDA terms used in the MARC 337 and 338 fields.

Catalogers also revised the GMD "electronic resource" for the consortial catalog. They agreed that this GMD should provide more granular information regarding its description and categorization since "electronic resource" was previously used to describe and categorize multiple format types of electronic origin. By deriving common terms such as "CDROM," "computer game," "video game," "electronic book," "electronic video," and "software," MSU catalogers created item-specific GMDs to allow users to easily disambiguate item types of various library materials that were similarly categorized as "electronic resource."

After catalogers revised legacy GMDs, the newly revised GMDs were mapped to the corresponding RDA 33X fields, and then to the item types to create a Word document table that MSU and MLP catalogers could use as a reference tool (see lib.msstate.edu/_assets/docs/mlp/MLP-RDA.pdf). This table featured a comprehensive list of mappings from the item type to 33X fields to the revised GMD. After revising GMDs for the MLP in December 2014, MSU Libraries distributed the Word document table mapping GMDs to item

\begin{tabular}{|c|c|c|c|}
\hline atlas & audiocassette & cartographic chart & cd recording \\
\hline cd-rom & $c d v$ & compact disc & dvd \\
\hline dvd-rom & $\begin{array}{l}\text { electronic resource } \\
\text { (cd-rom) }\end{array}$ & equipment & federal document \\
\hline floppy & globe & government document & graphic \\
\hline large print & laser disc & Ip & map \\
\hline map (tactile) & microfiche & microfilm & microopaque \\
\hline microprint & miscellaneous & newspaper & periodical \\
\hline photograph & record & serial & slide set \\
\hline $\begin{array}{l}\text { sound recording } \\
\text { (cd) }\end{array}$ & $\begin{array}{l}\text { sound recording } \\
\text { (Ip) }\end{array}$ & $\begin{array}{l}\text { sound recording (cas- } \\
\text { sette) }\end{array}$ & $\begin{array}{l}\text { sound recording } \\
\text { (compact disc) }\end{array}$ \\
\hline study print & us document & vhs & video single disc \\
\hline video-cd & videocassette & videodisc & videodisc (dvd) \\
\hline $\begin{array}{l}\text { videorecording } \\
\text { (dvd) }\end{array}$ & $\begin{array}{l}\text { videorecording } \\
\text { (vhs) }\end{array}$ & vsd & \\
\hline
\end{tabular}

Figure 3. Choosing Common Terms Options for a DVD

types and to the newly revised "common term" GMDs to each MLP cataloging unit.

\section{Testing the Process}

Prior to the conversion, Backstage developed an algorithm that extracted data values from the Leader, 007, 008, 245, 300,500 , and 538 fields in a bibliographic record to generate the RDA 33X fields and the newly revised GMDs. To test this algorithm, MSU Libraries and Backstage began implementing the conversion with a sample of bibliographic records that featured various item types including audio recordings, books, electronic resources, graphic novels, government documents, media, microforms, and photographs that were randomly selected from different libraries to ensure that there was appropriate representation from the consortium.

This sample was tested three times, and four catalogers spent an estimated 100 hours reviewing the tests. The first test file contained 793 bibliographic records and was sent to Backstage for processing in late October 2014, and was returned to MSU Libraries on October 30, 2014. This file tested the basic RDA enrichment algorithm provided by Backstage without changes to GMDs. In addition to basic RDA enrichment, the second test file also assigned the revised "common term" GMDs, and was received from Backstage on November 12, 2014. Catalogers reviewed these bibliographic records to ensure the modifications to Backstage's algorithm were accurately generating the newly revised GMDs.

For the third test file, the Computer Systems Assistant added fourteen bibliographic records to the initial 793 records, including three titles from the Early English books collection on microfilm, which features various "boundwith titles" (mlp.ent.sirsi.net/client/en_US/msstate/search/ detailnonmodal/ent:\$002f $\$ 002 \mathrm{fSD}$ ILS $\$ 002 \mathrm{f} 0$ \$002fSD_ ILS:2861289/ada?rt=CKEY||CKEY||false) and added eleven titles with mixed media item types such as books with 


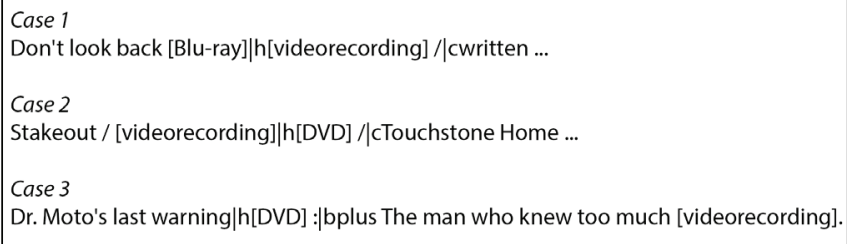

Figure 4. Examples of Two GMDs within Single Title Statement

accompanying CDs. By adding these bibliographic records, catalogers confirmed how Backstage's algorithm modified records for these complex formats. The third test file was returned to MSU Libraries on December 12, 2014. After reviewing results from the third test file, MSU Libraries decided to proceed with full RDA enrichment for the entire consortial catalog.

MSU's Computer Systems Assistant sent the full set of $1,800,186$ bibliographic records from the catalog to Backstage on December 15, 2014 to be processed. This set was returned to MSU Libraries on December 19, 2014 to be loaded into the online catalog. Due to the holiday recess, the loading process on the production server did not begin until January 6, 2015. The entire process took one week, and was not without issues. During this process, the catalog was unavailable for editing bibliographic records until all data was loaded on the production server. However, patrons could still access and use the catalog during this time.

\section{Findings}

Several months after the conversion, the authors systematically reviewed RDA-enriched records to check for accuracy, as they sought to identify, correct, and prevent future problems. Some of the inconsistencies found included incorrect 33X fields, multiple GMDs, and incorrect GMDs. Various keyword searches were conducted in the online catalog to identify GMD issues that may have occurred following RDA enrichment. Since Backstage processes and converts newly loaded records quarterly, the authors limited search results to retrieve titles loaded before September 30, 2015 to eliminate any identifiable post-enrichment errors that may have been corrected during subsequent Backstage processing.

The authors discovered many inconsistencies that resulted from cataloging errors, including the presence of two GMDs, only one of which was in MARC subfield $\$$ h. One particular example included a title statement with two GMDs, "Blu-ray" and "videorecording." Figure 4 illustrates three cases with Blu-ray or DVD titles in which two existing GMDs were provided within a single title statement.

MSU catalogers have yet to determine a consistent reason for two simultaneously occurring GMDs, but will correct these errors in the future. Catalogers identified this

\begin{tabular}{lc}
\hline Table 1. Search Results for Two Existing GMDs in Online Catalog \\
\hline Search Query & Titles Retrieved \\
"blu-ray" and "videorecording" & 41 \\
"DVD" and "videorecording" & 734 \\
"VHS" and "videorecording" & 24 \\
"CD" and "sound recording" & 219 \\
"audiocassette" and "sound recording" & 8 \\
"music CD" and "sound recording" & 11 \\
\hline
\end{tabular}

problem when conducting search queries using the catalog's "Advanced Search" page for previously used GMDs such as "videorecording" and "sound recording" and revised GMDs such as "DVD" and "music CD." Table 1 presents search results yielding two simultaneously existing GMDs, one obsolete and one revised term, within a single title statement.

Catalogers also discovered incorrect 33X fields, which they concluded were generated from Backstage's algorithm. Since Backstage extracted data from the 007 fields to generate the 33X fields, a record with either an incorrect 007 field or multiple 007 fields consequently produced incorrect 33X fields. For instance, if the item type was designated as a DVD, the first data element in the 007 field should have been coded as "v" while second data element should have been coded as "d." However, there were twenty-four records in which the second 007 data element was coded as "f," indicating the item is designated as a videocassette, which consequently generated the GMD "VHS."

Many of the records with this issue were created following earlier local policies, in which print and electronic versions of works were represented in the same bibliographic record. When the records were later separated to represent their specific format types, the 007 field was retained on the record that represented the print version of the work due to lack of appropriate bibliographic and item record maintenance. Problems with the 007 field data accuracy caused issues generating 33X fields and also with the OPAC's format display. When performing routine tasks in the ILS, serials and monographic catalogers discovered that GMDs and the format icons did not consistently match for every record, prompting them to review the OPAC for further display issues and inconsistencies.

After catalogers recognized that incorrect 007 field data could generate inaccurate GMDs and 33X fields in the bibliographic record and icons in the OPAC display, they initiated a policy change in the treatment of 007 fields. First, MSU catalogers must review the coded data element in the first 007 field of a bibliographic record and confirm whether it accurately categorizes and describes the primary format type of the work in hand. Second, should multiple 007 fields 


\author{
Songs of the Selma-Montgomery march [vinyl record]. \\ Author Seeger, Pete, 1919-2014. \\ Publication Date 1965 \\ Format $\checkmark$ Globe \\ Available: 1 \\ Copies: 1
}

Figure 5. Example of Title with Display Icon for Globe and GMD "vinyl record"

for nonfiction audiovisual materials than was previously available and to distinguish circulation periods for fiction audiovisual materials. However, this new item type, which was designed to include several different item format types including DVDs, VHS tapes, and CDs, was problematic when when MSU catalogers tried to revise GMDs for the consortia. Catalogers devised the GMD "nonfiction audiovisual" as a temporary solution

exist in one bibliographic record, catalogers must identify the 007 field that corresponds to the primary item type in hand and revise the order of the 007 fields to list the primary 007 field first. By reorganizing the 007 fields to list the primary item type as the first 007 field, the OPAC display generates the appropriate icon for an item type.

Another frequently occurring error was the presence of the 007 field for globes in bibliographic records for vinyl records, which was identified in 305 records. After discovering this error, catalogers searched the online catalog for all titles with this format and discovered 357 results, of which only one correctly represented globe as a resource. While the origin of this error remains unclear, catalogers will correct this issue as part of the ongoing cleanup. Figure 5 illustrates instances where the GMD "vinyl record" was present in a record with corresponding item type designated as "globe."

After catalogers discovered inconsistencies between 007 fields and GMDs, they coordinated with the acquisitions and systems departments to develop reports comparing information in the 007 field with the GMD. Each report was produced as an Excel spreadsheet and provided a listing of titles with 007 information and its corresponding GMDs. For instance, from 5,519 individual titles with the first two 007 subfield elements coded as "a" and " $\mathrm{j}$ " the assigned GMDs included eleven with "electronic book," sixty-four with "CD-ROM," thirty-four with "cartographic material," three with "graphic," 3,124 with "electronic resource," and 2,218 with "map."

Inconsistencies in the designation of certain item types also generated incorrect GMDs. While the majority of libraries in the consortium used the item types "audio-cd" for audiobooks and "music-cd" for music CDs, several libraries assigned inaccurate designations and used the item type "audio-cd" to categorize music CDs. Since GMDs were mapped to item types, all music CDs with a corresponding item type of "audio-cd" had a GMD of "audiobook" following the conversion. Similarly, one library system within the consortium used an item type designated as "NF-AV" for nonfiction audiovisual materials. This item type was established to enable catalogers to designate an extended circulation period and will reassess its value in the future. Although the online catalog currently includes an estimated 4,100 records with the GMD "nonfiction audiovisual," the location designation and icon provided help to clarify the corresponding item format. Figure 6 represents an online catalog record display with usage of the GMD "nonfiction audiovisual."

Following the conversion, the Cataloging Department worked closely with the Systems Department to generate reports reflecting conversion errors, including reports containing 007 fields and item types. Catalogers reviewed those reports to identify the most frequently occurring errors, to plan for subsequent online catalog maintenance, and to prevent errors in quarterly batch loading services from Backstage. Although catalogers initially ran bibliographic record reports and conducted searches to identify errors related to the conversion, unrelated errors were also discovered. For example, the authors discovered a significant batch of previously unreceived order records in the ILS, which were identified for future maintenance. As a result, the authors are currently coordinating with others at MSU Libraries to develop hands-on training sessions and enhance cataloging and acquisitions documentation for internal and MLP practices.

\section{Discussion}

In addition to developing training sessions, MSU Libraries catalogers continue to update local cataloging policies to establish consistent practices across the MLP. For instance, to prevent previously noted display errors originating from multiple 007 fields, all monographic catalogers met in October 2015 to establish a new policy, which involved analyzing all 007 fields in a bibliographic record prior to linking an item record. According to the new policy, only the 007 field correlating to a resource's primary format type are retained in the bibliographic record; all others are deleted or revised so that the primary 007 field information is listed first in the bibliographic record.

A more recent issue highlights inconsistent cataloging practices within the MLP that caused discrepancies between 
the 33X fields and GMDs following RDA enrichment. The authors discovered that several consortial libraries were not creating item records in the catalog for DVDs and other media types, which occurred when a large batch of records were loaded for an online streaming video service. Additionally, acquisition records lack item information until they are received, and bibliographic records for cancelled orders are not always deleted.

After identifying and analyzing issues that occurred post-enrichment, the authors concluded it was necessary to revise MLP cataloging procedural guidelines to ensure that each member library follows consistent practices for original and copy cataloging. A recent discovery involved creation of original bibliographic records for monographs that did not follow any established cataloging standard. After investigating this issue, it was revealed that two consortial libraries had created brief bibliographic records strictly for local usage and were not applying national cataloging standards. Such practices are problematic since there were pertinent RDA elements missing from the records, plus outdated GMDs. To address these issues and provide updated procedural guidelines, MSU Libraries hosted a training session on June 24, 2016 for MLP catalogers. The authors will also propose regular consortial catalog maintenance sessions for media resources to clean up metadata in the catalog.

While a cataloger can edit individual records manually, retrospective editing of large amounts of data is not ideal or cost-effective. If MSU Libraries pays a vendor for RDA enrichment services, it is logical that they want to limit maintenance post-enrichment. Therefore, it is important that all necessary bibliographic record elements be consistent for vendor-supplied RDA enrichment to be fully effective. Furthermore, without clear policies and procedural guidelines for catalogers to reference, inaccurate cataloging practices will likely continue despite vendor-supplied enrichment services.

\section{Conclusion}

As display and functionality of CMC information are still evolving, libraries have the opportunity to supplement this information locally with GMDs to best support their user needs. Although MSU Libraries displays the 33X fields in its OPAC, the clarity of this information is not easily interpreted by patrons. To remedy this issue, creating a vocabulary featuring more granular GMDs derived from "common terms" or item-specific information in the consortial catalog enabled MSU Libraries' catalogers to provide consistent and clean metadata necessary to support the user tasks of selection and identification, which was challenging with the legacy vocabulary of GMDs. While catalogers continue to assess the usefulness of the revised GMDs, patrons have expressed satisfaction with retaining GMDs in the online catalog. Moreover, supplying revised GMDs helps users to clarify the vocabularies used to represent RDA's CMC information displayed in the OPAC. For example, by providing the GMD "music CD," a user has more specific information regarding an item's format and the devices needed to access the content on the item, as compared to the ambiguous information found in the 33X fields such as "audio disc." From this RDA implementation project, MSU Libraries have identified a temporary solution to the limitation of its ILS display functions.

The authors believe that collaborating with the MLP and Backstage to standardize GMD and CMC processing before RDA conversion was the most effective action in providing clean and consistent metadata in the consortial catalog. Mapping item type designations was also useful in revising legacy GMDs. However, this RDA implementation project illustrates the importance of uniform cataloging practices in a consortial environment when considering working with a vendor to enrich or "hybridize" all the consortium's bibliographic records. Without uniform cataloging practices, a vendor-supplied conversion will not yield consistent results.

Furthermore, careful consideration should be taken in selecting a test sample of bibliographic records in the beginning stages of an enrichment process. Without a fully representative and accurate sample of bibliographic records, it is difficult to identify algorithmic problems that appear following batch conversions since there are a multitude of variables involved in the vendor's algorithm for GMD and CMC processing. From this, the authors concluded that bibliographic records must include consistent metadata prior to conversion in order to yield optimal results. By better understanding the vendor algorithm prior to conversion, catalogers 
and computer systems librarians can reduce errors that will allow for smooth RDA implementation.

\section{References}

1. Sevim McCutcheon, "RDA and the Reference Librarian: What to Expect from the New Cataloging Standard," Reference Librarian 53, no. 2 (2012): 125, https://doi.org/10.1080 /02763877.2011.607409.

2. "Dear Elsie," ILA Reporter 28, no. 6 (2010): 28, accessed October 30, 2016, http://www.ila.org/content/documents /Reporter_1210.pdf.

3. Jean Weihs and Lynne C. Howarth, "Designating Materials: From 'Germane Terms' to Element Types," Cataloging \& Classification Quarterly 45, no. 4 (2008): 3-24, https://doi .org/10.1300/J104v45n04_02.

4. Ibid.

5. Philip Hider, "A Comparison Between the RDA Taxonomies and End-User Categorizations of Content and Carrier," Cataloging \& Classification Quarterly 47, no. 6 (2009): 544-60, https://doi.org/10.1080/01639370902929755.

6. Weihs and Howarth, "Designating Materials," 5-6.

7. Ibid.

8. Dana M. Caudle and Cecilia Schmitz, "Keep It Simple: Using RDA's Content, Media, and Carrier Type Fields to Simplify Format Display Issues," Journal of Library Metadata 14, no. 3-4 (2014): 222-38, https://doi.org/10.1080/19386389.20 14.984572 .

9. Chris Oliver, Introducing RDA: A Guide to the Basics (Chicago: American Library Association, 2010), 50-51.

10. Caudle and Schmitz, "Keep It Simple," 229.

11. Oliver, Introducing RDA, 50-52.

12. Carol Ou and Sean Saxon, "Displaying Content, Media, and Carrier Types in the OPAC: Questions and Considerations," Journal of Library Metadata 14, no. 3-4 (2014): 239-54, https://doi.org/10.1080/19386389.2014.990846.

13. Michele Seikel and Thomas Steele, "How MARC Has Changed: The History of the Format and Its Forthcoming Relationship to RDA," Technical Services Quarterly 28, no. 3 (2011): 322-34, https://doi.org/10.1080/07317131.2011.57 4519 .

14. Ibid.
15. McCutcheon, "RDA and the Reference Librarian," 125.

16. OCLC, "336 Content Type," OCLC Bibliographic Formats and Standards, last modified August 22, 2016, http://www .oclc.org/bibformats/en/3xx/336.html.

17. OCLC, "337 Media Type," OCLC Bibliographic Formats and Standards, last modified August 25, 2016, http://www .oclc.org/bibformats/en/3xx/337.html.

18. OCLC, "338 Carrier Type," OCLC Bibliographic Formats and Standards, last modified August 22, 2016, http://www .oclc.org/bibformats/en/3xx/338.html.

19. Steven Bernstein, "Beyond Content, Media, and Carrier: RDA Carrier Characteristics," Cataloging \& Classification Quarterly 52, no. 5 (2014): 463-86, https://doi.org/10.1080/0 1639374.2014.900839.

20. Oliver, Introducing RDA, 17-23.

21. Terry Willan, "RDA in the Library System: Implementation and Beyond," Catalogue \& Index 163 (2011): 14-17.

22. Christopher Cronin, "From Testing to Implementation: Managing Full-Scale RDA Adoption at the University of Chicago," Cataloging \& Classification Quarterly 49, no. 7-8 (2011): 626-46, https://doi.org/10.1080/01639374.2011.616 263.

23. Caudle and Schmitz, "Keep It Simple," 222-38.

24. Roman S. Panchyshyn, "RDA Display and the General Material Designation: An Innovative Solution," Cataloging \& Classification Quarterly 52, no. 5 (2014): 487-505, https:// doi.org/10.1080/01639374.2014.902893.

25. Roman S. Panchyshyn and Amey L. Park, "Resource Description and Access (RDA) Database Enrichment: The Path to a Hybridized Catalog," Cataloging \& Classification Quarterly 53, no. 2 (2015): 214-33, https://doi.org/10.1080/0163937 4.2014 .946574 .

26. Richard Guajardo and Jamie Carlstone, "Converting Your E-Resource Records to RDA," Serials Librarian 68, no. 1-4 (2015): 197-204, https://doi.org/10.1080/0361526X.2015 .1025654 .

27. Backstage Library Works, "Common Terms: Level 2," accessed September 30, 2016, http://ac.bslw.com/mars/guide /RDA_Planning_Guide.pdf.

28. Backstage Library Works, "AACR2 GMD Terms Checked," accessed September 30, 2016, http://ac.bslw.com/mars/guide /RDA_Planning_Guide.pdf. 